\title{
«À LA FIN DE LA DECADENCE » : LA TRADUCTION DE LANGUEUR DE PAUL VERLAINE PAR ZENON PRZESMYCKI ET SON INFLUENCE SUR LA DEFINITION POLONAISE DU DECADENTISME EUROPEEN
}

\section{SŁOWA KLUCZOWE}

Zenon Przesmycki; Miriam; Paul Verlaine; Langueur; Niemoc; dekadentyzm; fin-de-siècle; koniec wieku; transfer kulturowy; translatologia; Kazimierz Wyka; Maria Podraza-Kwiatkowska; Andrzej Z. Makowiecki

\section{Le Plan de Paris. Définir la modernité}

Dans la deuxième moitié du XIX ${ }^{e}$ siècle, Paris devient le modèle de la ville moderne. La transformation de la capitale française entre 1852 et 1870 est un grand succès de la propagande du Second Empire, dont profitèrent Napoléon III ainsi que le baron Haussmann pour mener à bien la politique intérieure et étrangère (Pinkney 1958 : 1-29). Largement commentée par la presse nationale, la transformation même démontrait qu'il était possible de moderniser totalement une ville en une grande partie médiévale, qui choquait les Français et les étrangers par son étroitesse, sa mauvaise communication et son manque d'hygiène. En revanche, quelques dizaines d'années plus tard, les voyageurs visitant la capitale s'enthousiasmaient pour l'organisation parfaite de l'espace, les excellentes canalisations et ressentaient la vitesse de la ville qui se modernisait de façon dynamique.

Symbole le plus clair de la modernité du XIX ${ }^{\mathrm{e}}$ siècle, la photographie servit à soigneusement documenter les changements et permit à des cercles de plus en

Albert Kozik — mgr, Uniwersytet Warszawski, ul. Krakowskie Przedmieście 26/28, 00-927 Warszawa, e-mail; albertkozik@gmail.com; https://orcid.org/0000-0002-6813-4393 
plus larges de suivre ces métamorphoses. La modernité apparaissait à vue d'œil, et le sentiment que l'on pouvait l'observer et la ressentir se répandait dans toutes les couches de la société. Les réverbères, la canalisation ou les urinoirs publics constituaient ses réalisations physiques, et la possibilité de voir tous ces changements au moment précis où ils étaient fabriqués étaient le signe de sa présence. Les abondants reportages de presse sur les nouveautés techniques (comme l'utilisation de la lumière électrique pour éclairer les travaux nocturnes ${ }^{1}$ ) et les matériaux visuels, comme les dessins ou les photographies de Charles Marville, y contribuaient également.

Conformément au projet, la ville devait être transformée avec le soin le plus hygiénique pour cesser d'être un foyer de choléra, de pauvreté et de tendances révolutionnaires et devenir la magnifique capitale du grand empire. Ce ne fut qu'après 1870, qu'il fut possible d'organiser l'exposition universelle à Paris avec un panache sans précédent, et d'inscrire la ville dans l'histoire de la science et des techniques développées en France depuis $1789^{2}$. Parfaitement visible de tous les endroits parisiens, la Tour Eiffel devait démontrer toutes ces prouesses ${ }^{3}$.

Les images littéraires de Paris de la seconde moitié du XIX ${ }^{\mathrm{e}}$ siècle portent bien évidemment la marque de la vision de la modernité populaire à l'époque. Les récits mêlent souvent les représentations d'une ville se modernisant rapidement et mangeant en même temps ses habitants, d'une monstre et d'un salut, d'une promesse de paradis et de tombe. Ce réseau complexe et polysémique de métaphores nécessita des points de référence historiques et força en quelque sorte la recherche d'allégories dans le passé pour conceptualiser le développement de l'agglomération parisienne de façon appropriée. L'un des schémas fondamentaux fut donné par la ville antique de Rome dans sa période tardive, apparaissant aux artistes de la fin du XIX ${ }^{\mathrm{e}}$ siècle comme une représentation claire de la moralité " décadente ». Capitale de l'empire culturel de l'Europe, Paris devait nécessairement être comparé à la capitale de l'empire romain qui, elle aussi, dominait dans la culture de son temps. Les derniers siècles de l'empire romain sont considérés comme une époque d'affaiblissement et de marasme, de la même manière que la France fin de siècle joue un rôle de plus en plus limité dans l'arène politique internationale et est similairement décrite comme s'étant éloignée des idéaux moraux. Le progrès et la modernité ont généré de l'ennui et de la fatigue, exigeant des parallèles dans l'histoire du monde. Dans ce sens, les tendances "décadentes "

1 V. l'illustration de Jules Galdrau (Galdrau 1854 : 16).

2 V. le discours fait par le président Sadi Carnot justifiant l'organisation de L'Exposition universelle de 1889 (Carnot 1889 : 4).

3 La visibilité peut être d'ailleurs considérée comme une des catégories élémentaires avec lesquelles l'on pourrait décrire plusieurs expositions universelles françaises, comme le démontra d'une manière convaincante Timothy Mitchell, dans son analyse de l'exposition de 1852 (Mitchell 1991). 
de la seconde moitié du XIX siècle sont peut-être le résultat logique de l'image intensivement promue auparavant, celle d'une France-allégorie de la modernité ou une France-preuve de la grandeur de l'homme. Si l'on servait de la métaphore de l'empire dominant pour la décrire, elle devait forcément s'inverser et devenir le point de départ des images de chute de ce même empire.

Les chercheurs ont démontré à plusieurs reprises que le nouveau Paris était parfaitement adapté aux besoins de la consommation bourgeoise, mais les classes inférieures étaient plutôt exclues de sa structure (Murphy 2011 : 292). Le nouveau modèle de ville, projeté pour exposer les objets de la consommation publique, devait indéniablement renforcer l'analogie entre la Rome antique et Paris, comprise comme pars pro toto non seulement de la France, mais de toute l'Europe. Comme le remarque Philip Stephan, cette comparaison n'est d'ailleurs pas née dans la littérature des trente dernières années du XIX ${ }^{\mathrm{e}}$ siècle (Stephan 1974 : 19). La poésie française est comparée par Désiré Nisard à la poésie latine de l'âge d'argent déjà en 1834 dans L'Étude de moeurs et de critique sur les poètes de la décadence (Swart 1964 : 77) et, trois ans plus tard, Hugo, glorifiant Virgile, accentue les ressemblances entre Paris, Rome et Athènes. Paris est néanmoins plus lumineuse et plus bruyante que ses équivalents antiques :

[...] Lutèce, si petite au temps de tes Césars,

Et qui jette aujourd'hui, cité plaine de chars,

Sous le nom éclatant dont le monde le nomme,

Plus de clarté, qu'Athènes et plus de bruit que Rome

(Hugo 1909: 406)

C'est dans cette ambiance que Paul Verlaine publie son célèbre sonnet Langueur dans « Le Chat mort » le 26 mai 1883 (Stephan 1974 : 42). Il est reconnu comme la quintessence et le porte-drapeau du mouvement littéraire du " décadentisme ", au point qu'il est aujourd'hui inconcevable de définir l'état de la culture européenne de la fin du XIX ${ }^{\mathrm{e}}$ siècle sans celui-ci.

À l'époque, ce n'était point un texte isolé, mais plutôt le maillon d'un grand réseau de références métaphoriques, laborieusement entrelacées par les poètes (principalement parisiens d'ailleurs) de cette période. La comparaison entre Paris et Lutèce devint un élément typique de ce système des significations littéraires (Stephan 1974 : 40). Plusieurs textes ne tendent pas tant à esquisser des analogies, qu'à transposer et transformer de Paris en Rome impériale. C'est le cas d'Émile Goudeau dans son poème Les Affranchies: les charrettes de la capitale se transforment en esclaves affranchies et la ville du XIX siècle en ville antique (Goudeau 1913 : 15). Comme le grand Arc de Triomphe a été érigé à Paris, Napoléon ${ }^{\text {er }}$ devait forcément être césar, et les habitants de la ville - «les Romains 
de Paris ». Il ne s'agit aucunement d'une comparaison, puisque Goudeau va plus loin et confond la capitale de la France avec la capitale de l'Empire romain. Ainsi, Rome se réanime ou plutôt perdure sous une nouvelle forme, s'incarnant dans la nouvelle ville, dans le grand Paris du Second Empire.

Dans mon article, je souhaiterais analyser la manière dont Zenon Przesmycki dit Miriam, l'auteur de la seule traduction polonaise de Langueur, transpose l'imaginaire poétique de Verlaine dans la langue polonaise et assimile ce texte, qui était déjà connu, mais pas encore reconnu comme le porte-drapeau du décadentisme. Tout comme l'original, qui est progressivement devenu un des poèmes définissant l'époque, la traduction polonaise a joué un rôle déterminant dans la définition des mouvements littéraires et culturels de la fin du XIX ${ }^{\mathrm{e}}$ siècle en Pologne. Dans les travaux des spécialistes polonais de cette période, Verlaine fonctionne principalement en traduction, ce qui implique que le réservoir des termes utilisés par Przesmycki pour traduire le vocabulaire original constitue la principale source des catégories qui définissent le modernisme occidental (en particulier français). Je tenterai d'analyser les textes de chercheurs polonais tels que Kazimierz Wyka, Maria Podraza-Kwiatkowska et Andrzej Z. Makowiecki, qui ont synthétisé les phénomènes les plus importants de la culture européenne de la fin du XIX ${ }^{e}$ siècle.

Tout comme Paris qui devint le symbole de la modernité, et donc d'un certain modernisme, le poème de Verlaine - ou plutôt sa traduction - fut considéré comme l'incarnation de ce regard sur le monde et la littérature que l'on nomme "décadent ». Dans les synthèses scientifiques, Langueur et sa traduction Niemoc ont constitué des textes-clés, des points de référence et ont permis de naviguer entre les styles et les courants artistiques. La traduction de Przesmycki pouvait pourtant se s'avérer traîtresse, ce que n’ont pas remarqué les chercheurs. Comme toute traduction, le texte polonais est en effet une interprétation orientée d'une manière spécifique, ce que je me propose de démontrer ci-dessous.

\section{Où la langueur du soleil danse. Niemoc et Langueur}

Mis l'un juste à côté de l'autre, les deux poèmes se présentent comme suit :

Paul Verlaine

\section{Langueur}

\section{À Georges Courteline}

Je suis l'Empire à la fin de la décadence, Qui regarde passer les grands Barbares blancs

En composant des acrostiches indolents 
D'un style d'or où la langueur du soleil danse.

L'âme seulette a mal au cœur d'un ennui dense.

Là-bas on dit qu'il est de longs combats sanglants.

Ô n'y pouvoir, étant si faible aux vœux si lents,

Ô n'y vouloir fleurir un peu cette existence!

Ô n'y vouloir, ô n'y pouvoir mourir un peu!

Ah! tout est bu! Bathylle, as-tu fini de rire?

Ah! tout est bu, tout est mangé! Plus rien à dire!

Seul, un poème un peu niais qu'on jette au feu,

Seul, un esclave un peu coureur qui vous néglige,

Seul, un ennui d'on ne sait quoi qui vous afflige!

(Verlaine 1999 : 134)

Zenon Przesmycki

\section{Niemoc}

Jam Cesarstwo u schyłku wielkiego konania,

Które, patrząc, jak idą Barbarzyńce białe,

Układa akrostychy wytworne, niedbałe,

Stylem złotym, gdzie niemoc sennych słońc się słania.

Duszy, samiutkiej, mdło aż, w nudzie, co ochłania.

Skądciś tam wieści niosą walk olbrzymich chwałę.

O, nie móc, przez tę słabość, przez żądze tak małe,

$\mathrm{O}$, nie chcieć zaznać nieco tego falowania!

O, nie chcieć, o i nie móc umrzeć chociaż nieco!

Wszystko wypite! Ty tam, nie śmiej się z mych żali!

Wszystko, wszystko wypite! zjedzone! - Cóż daléj?

Tylko garść słabych wierszy, co ot w ogień lecą,

Tylko niewolnik nicpoń, co nie dba o pana,

Tylko ból jakiejś troski, co żre pierś, nieznana.

(Miriam 1921 : 193)

Le sonnet de Verlaine est conforme aux aspirations des artistes français de la seconde moitié du XIX ${ }^{e}$ siècle, désireux de réaliser une harmonie maximale dans la poésie. Connu aussi comme l'auteur de L'Art poétique, un manifeste pour une poésie musicale, le poète écrit Langueur dans l'intention de réaliser partiellement ce projet.

Dans le texte, la césure est rendue faible d'une manière surprenante et devient parfois presque entièrement inaudible. Verlaine suit de temps en temps la division classique de l'alexandrin en deux hémistiches $(6+6)$, mais il n’hésite pas à l'abandonner pour produire un effet rhétorique et mélodique:

Je suis l'Empire // à la fin // de la décadence,

Qui regarde passer // les grands Barbares blancs 
Le fragment sémantiquement le plus important du premier vers ( Je suis l'Empire ») est nettement séparé du groupe prépositionnel suivant ("à la fin »). L'haleine y est forcée par la logique syntaxique de la phrase, qui pousse le lecteur à faire une pause courte. Ainsi Verlaine isole la partie initiale du poème, la partie qui annonce son contenu, et oriente la compréhension du destinataire - c'est une stratégie avant tout rhétorique. Dans la plupart des vers il n'y a pas de césure après la troisième syllabe, et essayer de la prononcer détruit la logique syntaxique ainsi que la sémantique du poème. En lien avec cela, la matière tonique du texte devient irrégulière, parce que l'intonation n'est pas imposée par la structure de l'alexandrin, mais plutôt par ses intégrités syntaxiques et sémantiques :

Je suis l'Empire // à la fin // de la décadence,

Qui regarde / passer // les grands Barbares blancs

Ces opérations rendent le poème irrégulier au niveau rythmique, mais extraordinairement frappant au niveau sémantique. La partie mélodique du texte est fondée principalement sur des rimes qui correspondent à la signification des vers (par exemple, les centres des syllabes rimées, dans les deux premières strophes, sont les voyelles nasales : les unités phonétiques [dâs] (décadence-danse) et [lâ] (blancs-indolents)), et sur l'instrumentation poétique, elle aussi liée à la couche sémantique du texte (l'accumulation des consonnes occlusives voisées $[\mathrm{g}]$, [d] et [b], et encore la consonne fricative uvulaire voisée [в], au moment où l'on parle des «Barbares blancs »).

Toutes ces opérations phonétiques et rythmiques ont été brillamment traduites par Przesmycki dans Niemoc. Il a décidé de choisir le vers à treize syllabes (trzynastozgtoskowiec en polonais), propre à la poésie épique, qui peut être considéré comme l'équivalent de l'alexandrin français. Le traducteur a tenté de reproduire le rythme rompu de l'original en affaiblissant la césure par les parenthèses suivantes, ce qui donne un poème un peu plus chaotique mais fidèle aux intentions de l'auteur :

Jam Cesarstwo (/) u schyłku // wielkiego konania, Które, (/) patrząc, (/) jak idą // Barbarzyńce białe, Układa akrostychy // wytworne, / niedbałe,

Stylem złotym, / gdzie niemoc // sennych słońc się słania.

Bien que les divisions rythmiques du texte dépendent largement de la manière dont il est lu à haute voix, Przesmycki semble suggérer des pauses à des moments adaptés, en séparant adroitement les parenthèses suivantes par des virgules.

De plus, la matière phonétique du texte reflète les aspirations de Verlaine. En transposant la structure du sonnet italien employé dans le poème original, 
Miriam fait rimer de façon conséquente les syllabes contenant la consonne nasale palatale $[\mathrm{n}$ ] et les syllabes avec la consonne spirante labio-vélaire $[\mathrm{w}]$ dans les deux premières strophes, et ce, conformément à l'ambiance du texte. Afin d'accentuer la partie rythmique de la traduction, Przesmycki se sert d'une instrumentation poétique effective dans le dernier vers de la première strophe : il emploie une allitération et accumule les consonnes fricatives et nasalo-palatales ( Stylem złotym, gdzie niemoc sennych słońc się słania »).

Les changements les plus surprenants apparaissent surtout au niveau sémantique. En effet, Miriam a dû nécessairement inventer des équivalents des termes employés par Verlaine de manière à d'intégrer les concepts français dans la langue polonaise ; il est donc ici question d'un transfert culturel. Cela mène à des déplacements sémantiques très intéressants qui se reflètent dans l'ambiance et la signification générale du texte. La transposition d'un texte littéraire d'une langue à une autre entraîne toujours des changements considérables, résultant du transfert de notions culturelles (Espagne 2013).

Il faut tout d'abord analyser le titre de la traduction. En se servant du terme niemoc, Przesmycki ne traduit pas exactement le mot français langueur, qui a un champ sémantique similaire, mais ne correspond pas totalement à celui-ci. Le Dictionnaire de la langue française, dont la deuxième édition fut publiée par Émile Littré en 1883 (donc la même année que la publication de Langueur dans "Le Chat noir ») définissait ce mot comme suit :

$1^{\circ}$ État d'une personne affaiblie, malade. Maladie de la langueur. [...] $2^{\circ}$ Fig. Sorte d'affaiblissement moral et physique causé par les fatigues de l'esprit, par les peines de l'âme. $3^{\circ} \mathrm{Il}$ se dit, dans un sens analogue, de la passion de l'amour. $4^{\circ}$ État de l'âme qui se laisse aller à un état comparé à la langueur physique (Littré 1883 : III 249-250).

Mentionnées au début de la définition, quatre significations basiques de ce mot (sur six), décrivent parfaitement ses connotations sémantiques. Il s'agit donc d'un état d'affaiblissement ou de maladie, non seulement dans le sens physique (la première définition), mais encore dans le sens figuré (les définitions deux, trois et quatre). Le mot français conserve la signification de sa source latine : languor, -oris connote surtout "fatigue, abattement, langueur ", mais aussi " maladie, malaise, le mal d'amour [...] paresse, inaction" (Dictionnaire latinfrançais 1910 : 746). Toute la famille des mots basés sur le radical langu- a un sens de fatigue et d'inaction.

Le Trésor de la langue française fournit encore d'autres significations qui s'ajoutent au sens susmentionné. Les plus importantes suppléent la sémantique du mot : 
État d'âme mélancolique et rêveur qui rend nonchalant, sans énergie. [...] Caractère d'une chose (paysage, climat) dont la monotonie, la moiteur engendrent cet état d'âme (Trésor de la langue française).

L'original français suggère la persistance d'un état de fatigue, de mélancolie ; il évoque donc une image de maladie liée à l'inaction. Les définitions plus tardives, comme celle publiée dans le dictionnaire Micro Robert, accentuent même la durée particulière de cette condition :

$1^{\circ}$ État d'un malade dont les forces diminuent lentement $2^{\circ}$ Mélancolie douce et rêveuse (Robert 1984 : 602).

La Langueur est donc une situation dans laquelle " les forces diminuent lentement "; les exemples mentionnés par Littré en 1883 indiquent même que le caractère physique ou somatique de cet affaiblissement peut entraîner la mort. En polonais, Niemoc accentue beaucoup moins le caractère processuel de cette condition d'esprit, dans laquelle le sujet lyrique du poème de Verlaine se trouve. Le terme dénote plutôt l'aspect constant et invariable de cet état : il arrête le processus. Les expressions polonaises liées à ce terme préservent ce sens ("być zdjętym niemocą » - " être écrasé par l'impuissance »; " niemoc twórcza » - « l'impuissance créatrice »). Le mot n'a pas le sens de mélancolie. La traduction présente donc une réorientation légère de la sémantique, en accentuant plutôt le résultat ou le point d'arrivée que le processus ou la continuité de l'état d'affaiblissement. Ainsi, le premier changement peut être illustré de la manière suivante : affaiblissement, mélancolie (amoureuse), indifférence, maladie, fatigue, monotonie, amour $\rightarrow$ impuissance (créatrice).

Cependant, des conséquences beaucoup plus graves sont à noter suite à la décision de traduire l'expression "à la fin de la décadence ", qui finit le premier vers, par " u schyłku wielkiego konania » ("à la fin de la grande agonie »). Le mot " décadence " a, tout comme la langueur, une étymologie latine. Il vient de la langue latine vulgaire qui a donné naissance au terme decadentia, créé à partir du verbe decado, decadere (decido, decidere dans sa forme classique) signifiant « tomber, tomber sous les coups, périr, mourir, [...] déchoir, [...] succomber " (Dictionnaire latin-français $1910: 369)^{4}$. Ces connotations sont conservées dans décadence :

$1^{\circ}$ État de ce qui commence à choir, à tomber. [...] $2^{\circ}$ État de ce qui déchoit, au propre (Littré 1883 : II 974).

Dans les synonymes, Littré cite aussi des termes comme déclin et ruine. Verlaine souligne donc l'état d'un déclin progressif, d'une chute, le fait de tomber en

${ }^{4}$ Ce mot fut aussi préservé en anglais, ou il donna le terme decay "pourrir, se décomposer, s'avarier, pourrissement, décomposition ". 
ruine. Il lie la signification du mot décadence avec langueur qui est employé dans le titre. Miriam, quant à lui, se sert de l'expression " wielkie konanie » " la grande agonie "), qui est créée ad hoc et s'avère beaucoup plus forte, voire solennelle. Là, où le sujet lyrique se plonge dans la chute graduelle, l'inaction, l'ennui, la mélancolie, où il subit la fatigue et tombe en ruine, dans la traduction il est plutôt " écrasé par l'impuissance » et se trouve «à la fin de la grande agonie ".

Il est bien possible que l'interprétation de Przesmycki fût orientée par une signification additionnelle du mot décadence qui influença fortement la culture de la fin du XIX siècle (Stephan 1974 : 1-33). Peut-être Przesmycki désirait-il souligner le contexte (évident pour le lecteur français) dans lequel ce mot était utilisé et décida donc d'accentuer l'état "d'agonie ", compris dans un sens plus solennel et encore renforcé par l'épithète "grande ». Le terme polonais a plus le sens d'une mort douloureuse et difficile que celui de tomber en ruine, de mourir lentement et inactivement. Le deuxième transfert est donc le suivant : chute, déclin, tomber en ruine, déchoir $\rightarrow$ grande agonie.

Deux exemples susmentionnés illustrent à mon avis le désaccord principal qui existe entre la traduction et l'original. La version polonaise est rendue nettement plus solennelle, et ce, dès le début du texte, puisque « Je suis l'Empire » devient " Jam Cesarstwo ». Le traducteur se sert en effet d'une forme agglutinante, très soutenue en polonais. Miriam applique cette stratégie de façon très conséquente et donne aussi de nouveaux sens au texte verlainien, comme dans le cas de ce vers qui provient l'original :

Là-bas on dit qu'il est de longs combats sanglants.

Le vers qui lui correspond dans Niemoc est :

Skądciś tam wieści niosą walk obfitych chwałę.

"Longs combats sanglants ", qui suggèrent dans Langueur une situation désespérée et absurde, se transforment en "walk obfitych chwałę » (" la gloire des combats abondants »). Le traducteur non seulement utilise des expressions beaucoup plus imposantes, mais en plus il réarrange la signification du texte : la gloire n'a plus le sens d'absurde.

La situation semble comparable en ce qui concerne les figures de rhétoriques, qui sont très souvent inversées par Przesmycki :

Ah! tout est bu, tout est mangé ! Plus rien à dire!

devient dans sa traduction :

Wszystko, wszystko wypite! zjedzone! - Cóż daléj? 
Dans la version polonaise, le fragment est un peu plus dynamique, mais le changement fondamental vient du remplacement de la constatation définitive «Plus rien à dire " (exprimant plutôt la réalisation de l'état d'inaction et d'absurde) par la question "Cóż daléj ", qui est pleine de doute rhétorique. Cette tendance à la dramatisation du poème, faisant de lui un véhicule théâtral du sens, n'est point la seule dérogation à l'original que le traducteur s'est permis de faire. La plus intéressante est la réduction du sens dans la troisième strophe du sonnet.

Verlaine évoque la silhouette de Bathylle, qui est absente dans la traduction polonaise. La culture antique connaît au moins deux personnes de ce nom: le premier était un amant d'Anacréon, et le deuxième un acteur de l'époque d'Auguste, le bien-aimé de Mécène (Dizionario d'ogni mitologia 1809 : 286). En fait, on peut trouver les références à ce personnage dans les odes d'Anacréon (Moore 1800 : 72). Néanmoins, Verlaine n'est pas le premier à se servir de Bathylle dans la seconde moitié du XIX ${ }^{\mathrm{e}}$ siècle. Langueur est précédé par un autre poème intitulé Bathylle, écrit par Jean Lorrain et publié le $1^{\text {er }}$ juillet 1882 :

Au fond d'un bouge obscur, où boivent des marins,

Bathylle, le beau Thrace aux bras sveltes et pâles,

Danse au bruit de la flûte et des gais tambourins.

Ses pieds fins et nerveux, font claquer sur les dalles...

(Stephan 1974 : 41)

L'Atmosphère du poème se fonde sur le contraste entre l'image "d'un bouge obscur ", où les marins passent leurs nuits agitées, et la description du "beau Thrace ". Lorrain semble y lier l'idéalisation de la culture populaire comprise d'une manière spécifique avec le pressentiment que le vrai art peut justement naître dans de telles conditions provisoires. Le texte est rempli de métaphores et de comparaisons florales qui, avec les instruments musiques, constituent les principaux accessoires poétiques. Un rôle tout aussi déterminant est attribué aux énumérations des parties du corps (bras, pieds, talons, reins, ventre, œil, paume de la main), qui somatisent fortement le poème. Lorrain crée une image qui associe toutes les impressions sensuelles. La convention synesthétique doit peut-être accentuer l'artifice du travestissement et du maquillage de Bathylle qui, passant à la fin du XIX ${ }^{\mathrm{e}}$ siècle pour le modèle idéal du personnage transgressif, pouvait fonctionner entre les créations sexuelles masculines et féminines. C'est exactement cette idée qui est réalisée par les dessins célèbres d'Aubrey Beardsley.

Pour Verlaine, Bathylle était un double symbole. Il construisait avant tout un fil homo-érotique évident, à peine camouflé par le staffage antique. De plus, dans Langueur, le danseur joue le rôle du comique, auquel le sujet lyrique pose fièrement la question "As-tu fini de rire ? ». Dans la situation de décomposition 
culturelle, d'ennui et d'absurde, quand tout a été mangé, bu, consommé, tout rire devait apparaître comme vain. Il est aussi très significatif que Verlaine adresse son texte à Georges Courteline, le dramaturge comique français très célèbre à l'époque.

Przesmycki ne tient pas compte de tous ces sens qui ouvrent véritablement sur un deuxième niveau de compréhension du poème. Le vers qui contient la question rhétorique de Bathylle a la forme suivante dans le texte français :

Ah ! tout est bu, Bathylle, as-tu fini de rire?

En polonais, il est modifié comme suit :

Wszystko wypite! Ty tam, nie śmiej się z mych żali!

Le fait qu'un des moments les plus importants est transformé de manière surprenante «ty tam » (« toi, là-bas!») renverse totalement l'atmosphère solennelle bâtie avec soin dans la traduction. L'apothéose de l'art poétique, qui atteint l'acmé dans les derniers siècles de l'Empire romain pour ensuite tomber en ruine en raison de l'affaiblissement artistique et moral intérieur, est instantanément niée.

Les doutes et le désespoir du sujet lyrique, mêlés à l'éloge ("Je suis l'Empire... »), deviennent soudain le reproche d'une personne moquée pour ses regrets et qui, de plus, réagit par un cri prétentieux d'indignation. Ce moment de démystification, dont Przesmycki ne s'est peut-être pas lui-même rendu compte, ajoute un élément presque comique et détruit finalement le sens original. Tout cela a lieu bien avant la fin du poème, dans laquelle le traducteur se sert d'un mot qui rompt le registre de langue élevé, instauré depuis le début ("nicpoń » signifiant « chenapan ")

5 Pour mieux illustrer les différences entre la traduction et l'original, l'on pourrait se référer à deux tableaux qui représentent un sujet analogue : la situation de la Rome antique à la fin de son existence. Tous les deux peuvent illustrer le climat, la signification et la stylistique des poèmes. Le premier est la toile intitulée Les Romains de la décadence de 1847 de Thomas Couture. Les élites romaines, s'abandonnant à des plaisirs orgiastiques, représentent la culture antique qui tombe lentement en ruine, et dont le cadre de marbre entoure la scène. C'est une architecture froide (en accord avec les concepts d'art monochromatique de l'Antiquité), qui contraste avec la vivacité des corps des hommes et des femmes. Les idéaux d'harmonie et de symétrie sont engloutis de l'intérieur par la corruption morale et culturelle : voilà une vision parfaitement compatible avec l'ambiance du poème de Verlaine.

La chute de l'Empire romain dans la vision de Przesmycki est plutôt reflétée par le tableau de Thomas Cole intitulé Destruction qui provient du cycle The Course of Rome. Tel un colosse aux pieds d'argile, l'Empire s'effondre sous la pression des barbares; il manifeste encore sa résistance, mais perd totalement la bataille pour sa capitale. Embrasée par les flammes et écrasé par l'impuissance de sa propre grandeur, la ville n'est plus capable d'arrêter ce flot intarissable. Les édifices de Rome qui paraissent dans le lointain, pas encore touchés par le feu, semblent en même temps beaux 
Une traduction n'est jamais totalement équivalente au texte original. Le processus de lecture même et de transposition est une interprétation à plusieurs niveaux, souvent liée à la traduction de termes et de contextes non seulement absents dans la culture dans laquelle le poème est traduit, mais en plus souvent incompréhensibles pour celle-ci. Przesmycki crée donc un transfert culturel, tout en traduisant parfaitement la matière phonétique et en construisant son reflet rythmique et mélodique. Cependant, transférer le sens d'une langue à une autre est un travail particulièrement difficile et le niveau sémantique de la traduction ne peut pas vraiment correspondre à l'original.

Le texte de Przesmycki connut avec le temps une deuxième vie. En plus d'être un essai de traduction polonaise du texte le plus célèbre de Verlaine, il fut employé comme le réservoir des termes indispensables pour définir les principales tendances culturelles et artistiques de la fin du XIX siècle. Le traducteur n'a évidemment pas conçu sa traduction comme une préfiguration ou une incarnation de toute l'atmosphère intellectuelle et littéraire française de cette époque. L'acte de traduction était entièrement individuel, exprimant la volonté de créer une version polonaise d'un texte particulier. Dans ce sens, il devait s'agir d'une traduction comme beaucoup d'autres, si ce n'avait été la signification particulière attribuée à l'original et, par conséquent, à son équivalent polonais.

\section{La réception du poème par les chercheurs polonais et le rôle de la traduction dans ses analyses}

"Dans son célèbre poème Niemoc, Paul Verlaine écrivit : "Jam Cesarstwo na schyłku wielkiego konania” ». C'est ainsi que Maria Podraza-Kwiatkowska se réferre au poète français, quand elle analyse la lyrique pessimiste et décadente dans sa synthèse Literatura Mtodej Polski (Littérature de la Jeune Pologne). Elle emploie le premier vers de la traduction et le présente comme les mots de Verlaine en personne. La version polonaise est donc considérée comme une copie exacte de l'original. Aucun des chercheurs dont je souhaiterais décrire les thèses ne se penche explicitement sur le texte français en entier, se focalisant principalement sur la traduction polonaise (comme je le montrerai plus loin, Kazimierz Wyka ne cite qu'un fragment) pour éclairer le contexte du décadentisme français au lecteur polonais. Les poèmes de Verlaine et de Przesmycki sont utilisés suivant un schéma similaire dans toutes les synthèses. Ils ont donc une signification stratégique, car ils peuvent illustrer le climat intellectuel et culturel de l'époque.

et dépourvus de sens face à la destruction totale. Le tableau est tout aussi imposant et solennel que le texte de Przesmycki, mais son atmosphère n'est pas traduite dans l'ambiance du poème de Verlaine, qui associe principalement la chute à la décomposition. 
Dans le livre de Maria Podraza-Kwiatkowska, Niemoc n'est qu'un épisode dans le processus d'explication de la situation compliquée de l'Europe au tournant des $\mathrm{XIX}^{\mathrm{e}}$ et $\mathrm{XX}^{\mathrm{e}}$ siècles, mais il constitue aussi un exemple explicite de l'épuisement de la culture, la conscience "de surabondance d'elle-même, d'impossibilité de créer quelque chose d'entièrement nouveau (Nietzsche écrit : "Tout a été déjà fait", alors que Verlaine affirme : "Tout est bu, tout est mangé") " (PodrazaKwiatkowska 1992 : 44).

La situation est un peu différente dans l'ouvrage Mtoda Polska (Jeune Pologne) de Kazimierz Wyka. Le chercheur, dont la synthèse parut pour la première fois en $1977^{6}$, était un des premiers à proposer une conception aussi vaste de l'époque.

Dans le premier chapitre du deuxième tome, l'auteur tente de caractériser entièrement la Jeune Pologne et de construire un appareil terminologique qui pourrait servir à encadrer la littérature de cette période (Wyka 1987 : II 7-79). Bien sûr, une des notions principales à laquelle Wyka devait se référer était le mot "décadentisme ». Comme l'auteur le remarque à juste titre, la signification de ce terme diffère en polonais et en français. La notion française n’avait en général pas un sens défavorable à la fin du XIX ${ }^{e}$ siècle (et pouvait, comme le prouve le cas susmentionné de Nisard, produire des connotations positives), alors que le terme polonais eut rapidement mauvaise presse et fut principalement utilisé par les adversaires des nouvelles tendances dans la littérature (Wyka 1987 : II 11).

Wyka voulait examiner les origines de la réception négative des termes comme dekadencja ("décadence ") ou dekadent (" un décadent ") dans la langue polonaise. Pour ce faire, il se servit du poème de Verlaine afin de montrer les différences sémantiques entre les mots polonais et les mots français. Néanmoins, il provoqua un chaos terminologique, en confondant le titre du texte de Verlaine avec le dernier mot du premier vers, autrement dit langueur avec décadence :

Poetyckie określenie postawy dekadenckiej podawała np. pierwsza strofa głośnego sonetu Verlaine’a pt. Niemoc (tak Miriam transponuje tytuł Décadence) (Wyka $1987: 12$ ).

L'expression poétique de l'attitude décadente fut montrée par exemple dans la première strophe du fameux sonnet de Verlaine intitulé Niemoc (c'est la manière dont Miriam transpose le titre Décadence).

Wyka cite donc un faux titre et en tire les conclusions suivantes : " Miriam traduit judicieusement, en essayant d'éviter de comprendre la décadence comme une corruption ». Le chercheur ne se réfere pas directement à des dictionnaires

${ }^{6}$ Mtoda Polska fut publiée avec les essais de Wyka écrits en 1959 et intitulés Modernizm polski (Modernisme polonais), qui ne seront pas analysés dans cette thèse. 
français de l'époque (ou même contemporains), quoique il tente de reconstruire la signification du terme décadence par lui-même :

Oto po francusku décadent, décadence oznacza schyłek, chylenie się ku upadkowi, ale nie oznacza samego upadku, rozkładu z jego ujemną oceną, potworności, moralnej zgnilizny. Tego rodzaju zjawiska język francuski określa słowem déliquescence (Wyka $1987: 12)$.

En français, décadent et décadence signifient le déclin ou pencher vers sa fin, mais ils ne signifient pas la ruine en tant que telle, la corruption avec son sens péjoratif, la monstruosité, la pourriture morale. Les phénomènes de ce genre sont caractérisés par le mot déliquescence.

Il est difficile de juger sans ambiguïté cette analyse sémantique. Il ne fait aucun doute qu'à l'époque de Verlaine la notion de décadence n'avait pas - ou plutôt ne devait pas avoir - des connotations exclusivement négatives ${ }^{7}$. Toutefois, la manière dont Wyka définit le mot décadence en français n'est pas entièrement justifiée, puisqu'il clame qu'il s'agit seulement d'un processus consistant à " pencher vers sa fin ". Le dictionnaire de Littré mentionné précédemment souligne clairement le caractère synonymique des termes décadence et ruine. Le chercheur essayait peut-être de réorienter l'interprétation officielle, car à l'époque où il écrivit sa synthèse, la littérature de la Jeune Pologne était le plus souvent considérée comme sans valeur et non appropriée à des recherches sérieuses. Cité par l'auteur comme le synonyme le plus adapté de " corruption [...], monstruosité et pourriture morale ", le mot déliquescence est aussi, dans certain sens, discutable. Aujourd'hui, ce terme a certainement la signification susmentionnée, mais il n'est pas facile de déterminer la situation à la fin du XIX ${ }^{\mathrm{e}}$ siècle. Les dictionnaires français des années 1880, et donc contemporains de Verlaine, présentent des définitions étroitement chimiques : "phénomène offert par certains corps solides qui attirent l'humidité de l'air et se dissolvent » (Littré 1883: II 1037 ; Nouveau dictionnaire $1886: 224)$.

Pourtant, peu de temps après, Edmond de Goncourt décrit la mort de Verlaine en utilisant le terme déliquescence pour dépeindre l'état de son corps rongé par l'alcool, un corps qui, comme les substances chimiques, s'est dissous (Trésor de la langue française). Ce mot est employé pour décrire la décadence d'une civilisation ou sa culture à partir du début du XX siècle : il est très probable que la signification figurée existait au tournant des XIXe et $\mathrm{XX}^{\mathrm{e}}$ siècles,

7 Il est pourtant un fait que la plupart des dictionnaires du XIX ${ }^{e}$ siècle identifient avec le mot décadence les états du déclin progressif, de la mort ou de tomber en ruine, qui sont en général difficiles à considérer comme positifs. C'est par exemple la définition de ce terme selon Larousse de 1886, qui énumère les expressions tomber en ruine, en décadence comme les synonymes potentiels du verbe périr (Nouveau dictionnaire $1886: 571$ ). 
mais qu'elle n'était pas fixée, et certainement pas enregistrée par les lexicographes français dans leurs recherches. Une solution un peu plus sûre serait peut-être de se servir de synonymes aux significations bien établies dans années 1880 , comme abâtardissement, déchéance, dégradation, ou bien des termes suggérés par les dictionnaires de l'époque, comme affaiblissement ou déclin.

Par ailleurs, il semble très intéressant de noter que l'un des termes utilisés par Wyka pour définir décadence provient en fait de la traduction de Przesmycki ou plus exactement de la manière dont il transpose l'expression « à la fin de la décadence » ("u schyłku wielkiego konania »). Le chercheur adapte alors le réservoir terminologique de la version polonaise pour illustrer la signification du mot français. La situation est identique chez un autre chercheur polonais, Andrzej Z. Makowiecki, qui essaya par deux fois de définir le décadentisme dans ses travaux consacrés à la littérature de la Jeune Pologne. Il le fit dans le livre Mtoda Polska (Jeune Pologne), publié pour la première fois en 1981, puis dans le recueil d'articles édité par ses soins qui parut en 1992 dans le cadre du cycle Literatura polska (Littérature polonaise) (Makowiecki 1992 : 5-12).

Makowiecki, lui aussi, peut-être sous l'influence de Wyka, cite de manière erronée le titre du poème de Verlaine, et ce, dans ses deux analyses. Contrairement à son prédécesseur, il comprend le décadentisme comme un stade avancé de maladie ou de décomposition :

Owa „dekadencja” była więc rozumiana jako proces chorobowy, o objawach podobnych do „kryzysu” w zdrowiu chorego, poprzedzającego często jego śmierć, ale krótkotrwale łudzącego objawami polepszenia (Makowiecki 1987 : 6).

Une telle " décadence " était donc comprise comme un processus de maladie présentant des symptômes semblables à une « crise » dans la santé d'un malade, précédant souvent sa mort, bien que donnant une brève illusion d'amélioration.

Cette interprétation combine la signification des mots langueur et décadence. Elle est aussi plus proche de contextes réels dans lesquels on employait ces termes, contrairement aux explications de Kazimierz Wyka. La partie la plus frappante du discours de Makowiecki est celle qui vise à définir les notions de décadence et décadent mêmes. Le chercheur y emploie en fait deux mots de la traduction de Przesmycki :

Jedną z najwcześniejszych i do dziś będących w użyciu nazw odnoszących się do zjawisk literackich [...] jest termin „dekadentyzm” (od francuskiego „décadence” - 'schyłek, niemoc, przesilenie') (Makowiecki $1987: 5$ ).

Encore en usage aujourd'hui, le terme "décadentisme ", du français " décadence » - 'schyłek, niemoc, przesilenie', (« déclin, impuissance, crise »-A.K.) est [...] un des premiers noms à se référer à des phénomènes littéraires. 
Le rôle de la traduction de Przesmycki dans la définition des phénomènes caractéristiques de la littérature en France et en Pologne apparaît ici de manière explicite. Elle est, d'une part, un réservoir terminologique dont un chercheur peut se servir pour encadrer et définir les concepts utilisés pour nommer une époque et, d'autre part, un ensemble d'interprétations toutes prêtes, liées à ces concepts. Les mots de Miriam semblent aider les théoriciens à percer des phénomènes littéraires et culturels successifs. Non sans raison le poème est donc toujours cité au tout début des analyses. Il est un point de référence parfait et une sorte d'ancre, qui permet de raccrocher la littérature polonaise à la littérature occidentale. Il fonctionne donc comme une pars pro toto des grands courants de l'Europe (et, en particulier, de la France) ainsi que comme une clé d'interprétation pour le chercheur et le lecteur du décadentisme et, par extension du modernisme et de la Jeune Pologne.

Pour conclure les réflexions présentées ci-dessus, il ne fait aucun doute que la traduction de Langueur de Verlaine est essentielle, plus essentielle même que l'original, dans la littérature scientifique polonaise consacrée à la Jeune Pologne. Sa fonction peut être résumée comme suit :

1. Elle assimile à la littérature polonaise un texte représentatif de la culture française de la fin du XIX ${ }^{e}$ siècle, considéré comme le porte-drapeau des changements littéraires, artistiques et culturels de l'époque. Elle est, principalement, un transfert culturel puisqu'elle transporte toutes ces idées.

2. Conformément au point $\mathrm{n}^{\circ} 1$, elle est une pars pro toto de toute la culture de cette période.

3. Elle se présente comme un analogon de la littérature française, surmontant la barrière de l'étrangeté et polonisant Verlaine afin de le rendre compréhensible pour le lecteur polonais. Elle crée donc une situation inverse à la stratégie de Novalis qui aspirait à " rendre inhabituel ce qui était habituel " (Baxandall 2009: 38).

4. Elle constitue la source des termes scientifiques indispensables pour définir la littérature européenne occidentale de cette époque et la culture de la Jeune Pologne. Elle permet alors d'utiliser les mots qui s'y trouvent pour expliquer la sémantique des termes français.

5. Elle montre la manière historiquement contextualisée dont Verlaine était compris. Dans ce sens, elle est une voix de l'époque qui présente les interprétations du poète par la Jeune Pologne.

6. En lien avec les points ci-dessus, elle peut être employée comme une ancre d'interprétation, permettant de construire une narration scientifique sur la littérature, car elle fonctionne le plus souvent comme le texte de Verlaine lui-même, et non pas comme une traduction ; la plupart des scientifiques 
identifient en général l'original avec la version polonaise, comme s'ils fonctionnaient en dehors de la conscience des divergences sémantiques et culturelles contenue dans chaque texte.

La position du texte verlainien est évidente : c'est le symbole de certaines attitudes, changements et phénomènes sociaux. Les chercheurs l'utilisent comme un exemple, une preuve et un argument. Le texte a déjà sa propre légende. Mais la position de la traduction de Przesmycki est loin d'être claire. Le plus souvent, elle est instrumentalisée, traitée généralement comme le poème original, sans référence aux sources des mots utilisés par le traducteur. Les différences sèment la confusion entre les termes originaux et les termes traduits, ce que l'on observe dans les travaux scientifiques. Le poème de Przesmycki joue un rôle plus grand que celui que l'on imagine, et plus grand que ce que l'auteur lui-même aurait pu imaginer. La traduction n'est plus individuelle ; elle devient une clé pour comprendre les phénomènes de la littérature occidentale et polonaise, un ensemble des termes pratiques et, dans un sens plus général, la langue même de leur description.

\section{BIBLIOGRAPHIE}

Baxandall Michael. 2009. Prawda a inne kultury. Chrzest Chrystusa Pierra della Francesca. In: Perspektywy wspótczesnej historii sztuki. Antologia przektadów „Artium Quaestiones". Éd. Mariusz Bryl et al. Poznań : Wydawnictwo Naukowe Uniwersytetu im. Adama Mickiewicza. Pp. 27-68.

Carnot Sadi. 1889. Inauguration de l'exposition universelle de 1889. "Le Temps", $\mathrm{n}^{\circ}$ 10228. P. 4.

Dictionnaire latin-français. 1910. Éd. Louis Quicherat, Émile Chatelain. Éd. 2. Paris : Hachette.

Dizionario d'ogni mitologia e antichità. 1809. Éd. Girolamo Pazzoli, François Noel, Felice Romani et Antonio Peracchi. Vol. 1. Milano : Batelli.

Espagne Michel. 2013. La notion de transfert culturel. "Revue Sciences/Lettres ", ${ }^{\circ} 1$, http://rsl.revues.org/219 [7 juillet 2016].

Galdrau Jules. 1854. Travaux nocturnes des constructions de la rue de Rivoli, éclairés par la lumière électrique. «L'Illustration. Journal Universel », nº 605. P. 16.

Goudeau Émile. 1913. Les Affranchies. In : Idem, Poèmes à dire. Précédés d'une notice sur le livre et l'auteur par Jacques Ferny. Paris: P. Ollendorf. P. 15.

Hugo Victor. 1909. À Virgile. In : Idem, Euvres complètes: poésie. Vol. 2. Paris : Édition Paul Ollendorff et Albin Michel. S. 406.

Littré Émile. 1883. Dictionnaire de la langue française. Vol. 2 i 3. Paris : Hachette. 
Makowiecki Andrzej Z. 1987. Mtoda Polska. Éd. 2. Warszawa : Wydawnictwa Szkolne i Pedagogiczne.

Makowiecki Andrzej Z. 1992. Terminologia. Ramy chronologiczne okresu. In: Mtoda Polska. Éd. Andrzej Z. Makowiecki. Warszawa : Państwowe Wydawnictwo Naukowe. Pp. 5-12.

Miriam [Z. Przesmycki]. 1921. U Poetów. Przektady z poezji francuskiej, belgijskiej i wtoskiej XIX wieku. Warszawa : Wydawnictwo J. Mortkowicza.

Mitchell Timothy. 1991. Colonising Egypt. Éd. 2. Berkeley : University of California Press.

Moore Thomas. 1800. Odes of Anacreon. London : Printed for John Stockdale.

Murphy Kevin D. 2011. The Historic Building in the Modernized City: The Cathedrals of Paris and Rouen in the Nineteenth Century. "Journal of Urban History ", n 37 (2). Pp. 278-296.

Nouveau dictionnaire de la langue française. 1886. Éd. Pierre Larousse. Paris : Librairies Larousse et Boyer réunies.

Pinkney David H. 1958. Napoleon III and the Rebuilding of Paris. Princeton : Princeton University Press.

Podraza-Kwiatkowska Maria. 1992. Literatura Mtodej Polski. Warszawa : Państwowe Wydawnictwo Naukowe.

Robert Paul. 1984. Micro Robert : Dictionnaire du français primordial. Paris : Le Robert.

Stephan Philip. 1974. Paul Verlaine and the Decadence, 1882-90. Manchester : Manchester University Press.

Swart Koenraad W. 1964. The Sense of the Decadence in Nineteenth Century France. The Hague : M. Nijhoff.

Trésor de la langue française informatisé. Définitions des mots langueur et déliquescence, http://atilf.atilf.fr/dendien/scripts/tlfiv5/advanced.exe?8;s=100314195 [9 juillet 2016].

Verlaine Paul. 1999. One Hundred and One Poems by Paul Verlaine: A Bilingual Edition Translated by Norman R. Schapiro. Chicago : The University of Chicago Press.

Wyka Kazimierz. 1987. Mtoda Polska. Vol. 2. Kraków: Wydawnictwo Literackie. 
Albert Kozik

\title{
À LA FIN DE LA DÉCADENCE: ZENON PRZESMYCKI'S TRANSLATION OF PAUL VERLAINE'S LANGUEUR AND ITS INFLUENCE ON THE POLISH DEFINITION OF THE DECADENT MOVEMENT
}

\author{
(summary)
}

The article examines the most significant semantic changes and transpositions that can be found in Zenon Przesmycki's translation of the seminal sonnet Langueur written by Paul Verlaine in the second half of the nineteenth century. It closely analyzes the way in which the translation was used by Polish scholars of literary history. Having influenced the vision of culture nurtured by the so-called Decadent Movement in fin-de-siècle Europe, Verlaine's poem was then employed in numerous academic studies as an illustration of the philosophy and the attitude towards society that were dominant in the period under discussion. In Poland, Przesmycki's translation played an important role in defining the very term "decadent," as several influential researchers used it in their analyses in order to provide their readers with Polish equivalents of the original French terms. As a result, Przesmycki's version of Verlaine's sonnet, although intended just as a poetic translation, served a different purpose from the ones that its translator could have ever imagined. It helped define and describe the culture of "decadent" Europe as a whole. The terminology Przesmycki used to translate the French words, such as langueur and decadence ("niemoc" and "wielkie konanie" respectively), was thus introduced into the Polish scholarly debate on European modernism. Premised upon the idea of cultural transfer (transfert culturel), the analysis presented in the article helps determine the extent to which Przesmycki's translation shaped the Polish perception of fin-de-siècle Europe.

\section{KEYWORDS}

Zenon Przesmycki; Paul Verlaine; Langueur; Niemoc; the Decadent movement; fin-desiècle; cultural transfer; translation studies; Kazimierz Wyka; Maria Podraza-Kwiatkowska; Andrzej Z. Makowiecki 